This item was submitted to Loughborough's Research Repository by the author.

Items in Figshare are protected by copyright, with all rights reserved, unless otherwise indicated.

\title{
Prevention, diagnosis and treatment of the overtraining syndrome: Joint consensus statement of the European College of Sport Science (ECSS) and the American College of Sports Medicine (ACSM)
}

\section{PLEASE CITE THE PUBLISHED VERSION}

http://dx.doi.org/10.1080/17461391.2012.730061

\section{PUBLISHER}

Taylor \& Franics Ltd (@ 2013 European College of Sport Science)

\section{VERSION}

AM (Accepted Manuscript)

\section{PUBLISHER STATEMENT}

This work is made available according to the conditions of the Creative Commons Attribution-NonCommercialNoDerivatives 4.0 International (CC BY-NC-ND 4.0) licence. Full details of this licence are available at: https://creativecommons.org/licenses/by-nc-nd/4.0/

\section{LICENCE}

CC BY-NC-ND 4.0

\section{REPOSITORY RECORD}

Meeusen, Romain, Martine Duclos, Carl Foster, Andrew Fry, Michael Gleeson, David C. Nieman, John Raglin, Gerard Rietjens, Jürgen Steinacker, and Axel Urhausen. 2019. "Prevention, Diagnosis and Treatment of the Overtraining Syndrome: Joint Consensus Statement of the European College of Sport Science (ECSS) and the American College of Sports Medicine (ACSM)". figshare. https://hdl.handle.net/2134/17104. 


\section{Prevention, diagnosis and treatment of the Overtraining Syndrome}

Joint consensus statement of the European College of Sport Science (ECSS) and the American College of Sports Medicine (ACSM)

Romain Meeusen, Belgium (chair)

Martine Duclos, France

Carl Foster, USA

Andrew Fry, USA

Michael Gleeson, UK

David Nieman, USA

John Raglin, USA

Gerard Rietjens, The Netherlands

Jürgen Steinacker, Germany

Axel Urhausen, Luxembourg

Axel Urhausen :

Centre de l'appareil locomoteur, de médecine du sport et de prévention, Centre hospitalier of Luxemburg and CRPsanté, Luxembourg

urhausen.axel@chl.lu

Michael Gleeson :

School of Sport, Exercise and Health Sciences, Loughborough University, Loughborough, Leicestershire, LE11 3TU, UK

m.gleeson@lboro.ac.uk

Romain Meeusen :

Vrije Universiteit Brussel dept. Human Physiology \& Sports Medicine, Brussels, Belgium

rmeeusen@vub.ac.be

Gerard Rietjens :

Training Medicine and Training Physiology, Royal Netherlands Army, Utrecht The Netherlands

Vrije Universiteit Brussel dept. Human Physiology \& Sports Medicine, Brussels Belgium

gerardrietjens@gmail.com

Martine Duclos :

Department of Sport Medicine and Functional Exploration, University Hospital CHU G. Montpied, INRA, UMR 1019, UNH, CRNH Auvergne, University of Auvergne, F-63000 Clermont-Ferrand, France mduclos@chu-clermontferrand.fr

Jurgen Steinacker :

Sektion Sport- und Rehabilitationsmedizin, Universitätsklinikum UIm, Universität UIm, 89075 Ulm, Germany

juergen.steinacker@.uni-ulm.de

John Raglin :

Department of Kinesiology, Indiana University, Bloomington, IN, USA

raglinj@indiana.edu

Carl Foster :

Department of Exercise and Sport Sciencce, University of Wisconsin-La Crosse, La Crosse, WI, USA 
cfoster@uwlax.edu

David Nieman :

Department of Health, Leisure and Exercise Science, Applacian State University, Boone, NC, USA niemandc@appstate.edu

Andrew Fry :

Department of Health, Sport and Exercise Science, University of Kansas, Lawrence, KS, USA

acfry@ku.edu 
Introduction

The goal in training competitive athletes is to provide training loads that are effective in improving performance. During this process athletes may go through several stages within a competitive season of periodised training. These phases of training range from insufficient training, during the period between competitive seasons or during active rest and taper, to "Overreaching" (OR) and "Overtraining" (OT) which includes maladaptations and diminished competitive performance. Literature on "Overtraining" has increased enormously; however, the major difficulty is the lack of common and consistent terminology as well as a gold standard for the diagnosis of overtraining syndrome.

In 2006 the ECSS published its consensus statement on Overtraining (Meeusen et al. 2006). We decided to write an update and to ask the American College of Sports Medicine (ACSM) to provide input in this paper so that this can be considered as a mutual 'consensus statement' of both international organisations. In this "consensus statement" we will present the current state of knowledge on the Overtraining Syndrome (OTS) going through its definition, diagnosis, treatment, and prevention.

\section{Definition}

Successful training must involve overload but also must avoid the combination of excessive overload with inadequate recovery. The process of intensifying training is commonly employed by athletes in an attempt to enhance performance. As a consequence the athlete may experience acute feelings of fatigue and decreases in performance as a result of a single intense training session, or an intense training period. The resultant acute fatigue, after an adequate rest period can be followed by a positive adaptation or improvement in performance and is the basis of effective training programmes. However, if the balance between appropriate training stress and adequate recovery is disrupted, an abnormal training response may occur and a state of "Overreaching" may develop. Beyond this, the evidence for a supercompensation effect after deliberate periods of intensified training is not abundant.

Many recent papers have referred to the work of Kreider et al. (1998) for the definition of OT \& OR.

- Overreaching : an accumulation of training and/or non-training stress resulting in short-term decrement in performance capacity with or without related physiological and psychological signs and symptoms of maladaptation in which restoration of performance capacity may take from several days to several weeks.

- Overtraining : an accumulation of training and/or non-training stress resulting in long-term decrement in performance capacity with or without related physiological and psychological signs and symptoms of maladaptation in which restoration of performance capacity may take several weeks or months.

As stated by several authors (Budgett et al. 2000, Halson \& Jeukendrup 2004) these definitions suggest that the difference between OT \& OR is the amount of time needed for performance restoration and not the type or duration of training stress or degree of impairment. These definitions also imply that there may be an absence of psychological signs associated with the conditions. As it is possible to recover from a state of OR within a 2-week period (Halson et al. 2002, Jeukendrup et al. 
1992, Kreider et al. 1998, Steinacker et al. 2000), it may be argued that this condition is a relatively normal and harmless stage of the training process. However, athletes who are in an 'overtrained' state may take months or possible years to completely recover.

The difficulty lies in the subtle difference that might exist between extreme overreached athletes and those having an "Overtraining Syndrome" (OTS). The possibility also exists that these states (OR/OTS) show different defining characteristics and that the overtraining continuum may be an oversimplification.

To avoid misconception of terminology we here outline the terms OR, OT and the OTS based on the definitions used by Halson \& Jeukendrup (2004) and Urhausen \& Kindermann (2002). In these definitions "Overtraining" is used as a 'verb', a process of intensified training with possible outcomes of short term Overreaching (functional OR); extreme Overreaching (non-functional OR); or the Overtraining Syndrome (OTS). By using the expression 'syndrome' we emphasize the multifactorial aetiology and acknowledge that exercise (training) is not necessarily the sole causative factor of the syndrome.

Overreaching is often utilised by athletes during a typical training cycle to enhance performance. Intensified training can result in a decline in performance; however, when appropriate periods of recovery are provided, a 'Supercompensation' effect may occur with the athlete exhibiting an enhanced performance compared to baseline levels. This process is often used when going on a 'training camp', and will lead to a temporary performance decrement, which is followed by improved performance. In this situation, the physiological responses will compensate the training related stress (Steinacker et al. 2004). This form of short term "Overreaching" can also be called "Functional Overreaching". When this 'intensified training' continues, the athletes can evolve into a state of extreme Overreaching or "Non-Functional Overreaching", that will lead to a stagnation or decrease in performance which will not resume for several weeks or months. However, eventually these athletes will be able to fully recover after sufficient rest. "Non-Functional Overreaching" emphasizes that the evolution on the "overtraining continuum" is not only "quantitatively" determined (i.e., by the increase in training volume) but that also "qualitative" changes occur (e.g., signs and symptoms of psychological distress and/or endocrine disturbances). This is in line with the classical concept of "sympathetic versus parasympathetic OTS" (Israel 1976), and recent neuroendocrine findings using a double exercise test (Meeusen et al. 2004, 2010).

In figure 1 the different stages that differentiate normal training from OR (functional and nonfunctional OR) and from the OTS are presented. Training can be defined as a process of overload that is used to disturb homeostasis which results in acute fatigue leading to an improvement in performance. When training continues or when athletes deliberately use a short term period (e.g., training camp) to increase training load they can experience short term performance decrement, without severe psychological, or lasting other negative symptoms. This Functional OR (or short term OR) will eventually lead to an improvement in performance after recovery. However, when athletes do not sufficiently respect the balance between training and recovery, Non-Functional OR (extreme OR) can occur. At this stage the first signs and symptoms of prolonged training distress such as performance decrements, psychological disturbance (decreased vigour, increased fatigue), and hormonal disturbances will occur and the athletes will need weeks or months to recover. Several confounding factors such as inadequate nutrition (energy and/or carbohydrate intake), illness (most 
commonly upper respiratory tract infections, URTI), psychosocial stressors (work-, team-, coach-, family- related) and sleep disorders may be present. At this stage the distinction between NonFunctional OR and OTS is very difficult and will depend on the clinical outcome and exclusion diagnosis. The athlete will often show the same clinical, hormonal and other signs and symptoms. Therefore, the diagnosis of OTS can often only be made retrospectively when the time course can be overseen. A keyword in the recognition of OTS might be 'prolonged maladaptation' not only of the athlete, but also of several biological, neurochemical, and hormonal regulation mechanisms.

\section{$\Rightarrow$ Insert FIGURE 1 here}

The borderline between optimal performance and performance impairment due to "OTS" is subtle. This applies especially to physiological and biochemical factors. The apparent vagueness surrounding OTS is further complicated by the fact that the clinical features are varied from one individual to another, and are non-specific, anecdotal and numerous.

Diagnosis

Although in recent years the knowledge of central pathological mechanisms of the OTS has significantly increased there is still a strong demand for relevant tools for the early diagnosis of OTS. The OTS is characterised by a "sports-specific" decrease in performance together with disturbances in mood state. This underperformance persists despite a period of recovery lasting several weeks or months. Importantly, as there is no diagnostic tool to identify (e.g., rule in) an athlete as suffering from OTS, the solution to the differential diagnosis can only be made by excluding all other possible influences on changes in performance and mood state. Therefore, if no explanation for the observed changes can be found, OTS is diagnosed. Early and unequivocal recognition of OTS is virtually impossible because the only certain sign is a decrease in performance during competition or training. The definitive diagnosis of OTS always requires the exclusion of an organic disease, e.g., endocrinological disorders (thyroid or adrenal gland, diabetes), iron deficiency with anaemia, or infectious diseases (including myocarditis, hepatitis, glandular fever). Other major disorders or feeding behaviours such as anorexia nervosa and bulimia should also be excluded. However, it should be emphasised, that many endocrinological and clinical findings due to OR and OTS can mimic other diseases. The borderline between under- and over-diagnosis is very difficult to judge.

In essence, it is generally thought that symptoms of OTS, such as fatigue, performance decline, and mood disturbances, are more severe than those of OR. However, there is no scientific evidence to either confirm or refute this suggestion. Hence, there is no objective evidence that the athlete is indeed suffering from the OTS. Additionally, in the studies that induced a state of OR, many of the physiological and biochemical responses to the increased training were highly variable, with some measures in some studies demonstrating changes and others remaining unaltered, most likely, because conditions and the degree of OR and OTS differ and were not comparably described. This is also probably because the signs and symptoms of OTS are individual and it is not feasible and certainly unethical to excessively train an athlete in such a way that he/she will develop the OTS. Therefore, prospective studies are lacking and only few data exist on the OTS.

One approach to understanding the aetiology of the OTS involves the exclusion of organic diseases or infections and factors such as dietary caloric restriction (negative energy balance) and insufficient carbohydrate and/or protein intake, iron deficiency, magnesium deficiency, allergies, etc. together 
with identification of initiating events or triggers. One of the most certain triggers is a training error resulting in an imbalance between load and recovery. Other possible triggers might be the monotony of training, too many competitions, personal and emotional (psychological) problems and emotional demands of occupation. Less commonly cited possibilities are sleep disturbance, altitude exposure and exercise-heat stress. However, scientific evidence is not strong for most of these potential triggers. Many triggers such as glycogen deficiency or infections may contribute to OR or the OTS but might not be present at the time the athlete presents to a physician. Furthermore, identifying these possible initiating events has not revealed the causative mechanism(s) of the OTS. Consequently, some scientists have suggested that the OTS be renamed as the unexplained underperformance syndrome (Budgett et al. 2000) which focuses on the key symptom of underperformance in the OTS rather than on the mechanisms. This terminology has not been widely adopted outside the UK.

Athletes and the field of sports medicine in general would benefit greatly if a specific, sensitive simple diagnostic test existed for the diagnosis of the OTS. At present no test meets this criterion, but there certainly is a need for a combination of diagnostic aids to pinpoint possible markers for the OTS. Especially there is a need for a detection mechanism for early triggering factors.

Increased training loads as well as other chronic stresses can influence the neuroendocrine system chronically. However, at this time it is not yet clear which mechanism eventually leads to the OTS. Probably because of this, and because there are several possible hypotheses, a number of recent review articles have focused on hypothetical explanations for the mechanism behind the OTS. Although these theories have potential, until more prospective studies are carried out where a longitudinal follow up of athletes (who may develop the OTS) is performed, or specific diagnostic tools are developed, these theories remain speculative.

Prevalence

It is difficult to give exact prevalence figures on NFOR/OTS merely because not all studies clearly indicate the time frame of data collection. Survey research involving collegiate swimmers and other endurance athletes who completed a training monocycle report a rate of NFOR/OTS of approximately 10\% (Range: 7-21\%) (Raglin \& Wilson, 2000). Higher rates have been reported in other studies but these values are likely inflated by merging cases of FOR, NFOR and OTS. The risk of NFOR/OTS becomes compounded over the course of an athlete's career; survey studies of elite runners report $60 \%$ of females and $64 \%$ of males indicate experiencing at least one previous episode of OTS, with a career rate of $33 \%$ in non-elite adult runners (Morgan et al. 1987b; 1988b). Similar career rates of OTS have been reported by young athletes including a 34.6\% rate among 231 (age range: 13-18) age-group swimmers from four countries, with OTS being most common among faster performers (Raglin et al. 2000), and a 37\% rate in 272 Swedish high school junior national athletes assessed across 16 different sports (Kenttä et al. 2001). Retrospective techniques can be prone to bias or inaccurate recall, but a recent longitudinal study of British age-group swimmers found $29 \%$ had developed NFOR/OTS at least once, with the risk positively related to skill level (Matos et al. 2011). These findings reinforce both the growing risk of OTS for young athletes and the utility of retrospective methodologies in OTS research.

Moreover, there is evidence that athletes who have developed the OTS are at a heightened risk of relapse. In a study of U.S. collegiate swimmers, it was found $91 \%$ of the swimmers who developed 
OTS during their first collegiate training season were diagnosed with OTS again in one or more of the following three years of training. In contrast, only $34 \%$ of swimmers free of OTS during their first year of collegiate swimming had a later diagnosis of OTS (Raglin, 1993).

This interindividual variation in the risk for NFOR/OTS has been observed in athletes who undergo the same overload training. In a study of 13 competitive swimmers who completed 10 days of intensified training at the same volume and relative intensity $\left(8,970\right.$ m.day ${ }^{-1}$, at $\left.94 \% \mathrm{VO}_{2 \max }\right)$, seven swimmers successfully completed the required training regimen but three others had difficultly completing the training requirements, and these athletes had significantly higher levels of POMS mood disturbance (Morgan et al., 1988a) and lower levels of muscle glycogen (Kirwan et al. 1988). Another three swimmers were so severely affected by the training that they had to be dropped from the study.

It remains unclear whether these findings indicate some individuals are particularly predisposed to developing the OTS when exposed to overload training or whether succumbing to the OTS raises the risk of relapse. Some tests of potential psychological factors have been conducted and have not found the risk of OTS to be mediated by intrinsic motivation (Raglin \& Morgan 1994), hardiness, or optimism (Wilson \& Raglin 2004).

\section{Assessment of Overtraining}

The OTS reflects the attempt of the human body to cope with physiological and other stressors. Several studies have revealed that the OTS represents the sum of multiple life stressors, such as physical training, sleep loss, exposure to environmental stresses (e.g., exposure to heat, high humidity, cold, high altitude), occupational pressures, change of residence and interpersonal difficulties. Thus the OTS can be understood partly within the context of the General Adaptation Syndrome (GAS) of Seyle (1936). Concomitant to this "stress-disturbance" the endocrine system is called upon to counteract the stress situation. The primary hormone products (adrenaline, noradrenaline and cortisol) all serve to redistribute metabolic fuels, maintain blood glucose, and enhance the responsiveness of the cardiovascular system. Repeated exposure to stress may lead to altered responsiveness to subsequent stressful experiences depending on the stressor as well as on the stimuli paired with the stressor, either leading to an unchanged or increased or decreased neurotransmitter and receptor function. Behavioural adaptation (neurotransmitter release, receptor sensitivity, receptor binding etc.) in higher brain centres will certainly influence hypothalamic output (Lachuer et al. 1994). Lehmann et al. (1993a) introduced the concept, that hypothalamic function reflects the state of OR or the OTS because the hypothalamus integrates many of the stressors. It has been shown that acute stress not only increases hypothalamic monoamine release, but consequently corticotrophic releasing hormone (CRH) and adrenocorticotrophic hormone (ACTH) secretion (Shintani et al. 1995). Chronic stress and the subsequent chronically elevated adrenal glucocorticoid secretion could play an important role in the desensitisation of higher brain centres' response to acute stressors, since it has been shown that in acute and chronic stress the responsiveness of hypothalamic CRH neurons rapidly falls (Barron et al. 1985, Lehmann et al. 1993b, Cizza et al. 1993, Urhausen et al. 1998a).

The lack of definitive diagnostic criteria for the OTS is reflected in much of the 'overreaching' and 'overtraining' research by a lack of consistent findings. There are several criteria that a reliable marker for the onset of the OTS must fulfil: the marker should be sensitive to the training load and 
ideally, be unaffected by other factors (e.g., diet, chronobiological rhythms). Changes in the marker should occur prior to the establishment of the OTS and changes in response to acute exercise should be distinguishable from chronic changes. Ideally, the marker should be relatively easy to measure with a quick availability of the result, not too invasive (e.g., repeated venous blood samplings are not well accepted) and not too expensive. Ideally the marker should be derived at rest, from submaximal or standardised exercise of relatively short duration in order not to interfere with the training process. However, none of the currently available or suggested markers meets all of these criteria.

\section{BIOCHEMISTRY \& HORMONES}

Biochemistry

In prolonged training glycogen stores get close to full depletion, glycogenolysis and glucose transport are downregulated in muscle and liver as well as the liver production of IGF-1, and catabolism is induced. Although this is one of the likely triggers of OTS, muscle glycogen is typically normal when athletes are examined (Snyder 1999). Blood glucose is also not typically altered (Urhausen et al. 1998b). Resting blood glucose / insulin ratio may indicate mild insulin resistance (Steinacker et al. 2004).

Blood lactate measurements can be dependent on the actual training status of the individual. Other factors that are equally important when discussing changes in blood lactate concentrations are the glycogen status and possible decreases in muscle and liver stores due to increased training. One almost consistent overall finding, at least in endurance and strength-endurance athletes having the OTS, is a diminished maximal lactate concentration while submaximal values remain unchanged or slightly reduced (Urhausen \& Kindermann 2002).

Individually increased circulating levels of Creatine Kinase (CK), which especially reacts to eccentric and unaccustomed exercise with elevations lasting from several days to up to a little over one week, and/or urea measured under standardised conditions at rest (Urhausen \& Kindermann 1992), may provide information concerning an elevated muscular and/or metabolic strain (Urhausen et al 1989a), but they are not suitable to indicate an OR or OTS state (Urhausen et al. 1998a). Under glycogen depleted compared to carbohydrate loaded condition, serum urea increases during $1 \mathrm{~h}$ cycling at $61 \%$ VO2max but also before and $4 \mathrm{~h}$ after exercise (Lemon \& Mullin 1980). After one single eccentric strength exercise leading to a nearly 10 -fold maximal CK increase with a weak significant correlation to the isometric strength loss, the positive response to concentric strength training was significantly delayed for several weeks (Folland et al. 2001).

After 2 weeks of OR with short-term decline of performance and mood state, plasma CK (as well as glutamate) showed a significant and urea a tendency to increase before normalizing after 2 weeks of regenerative training in 8 moderately well trained cyclists (Halson et al. 2003) .

The concentration of plasma glutamine has been suggested as a possible indicator of excessive training stress (Rowbottom et al. 1995). However, not all studies have found a fall during periods of increased training and overtraining (Walsh et al. 1998) and altered plasma glutamine concentrations 
are not a causative factor of immunodepression in OTS, while other authors rather propose the glutamine/glutamate ratio as an indicator of OR (Smith \& Norris 2000; Coutts et al. 2007a).

Although most of the blood parameters (e.g., blood count, C-Reactive Protein, erythrocyte sedimentation rate, $\mathrm{CK}$, urea, creatinine, liver enzymes, glucose, ferritin, sodium, potassium, etc. ) are not capable of detecting OR or the OTS, they are helpful in providing information on the actual health status of the athlete, and therefore useful in the "exclusion diagnosis".

\section{Problems with biochemistry testing}

- Lactate differences are sometimes subtle (lying within the measuring error of the apparatus) and depend on the modus of the exercise test used

- No lactate changes reported in strength athletes

- Glutamine may fall with increased training load but low plasma glutamine concentration is not a consistent finding in OTS

\section{Hormones}

For several years it has been hypothesised that a hormonal mediated central dysregulation occurs during the pathogenesis of the OTS, and that measurements of blood hormones could help to detect the OTS (Lehmann et al. 1993b, Fry et al. 1991, Fry \& Kraemer 1997; Kuipers \& Keizer 1988; Urhausen et al. 1995, 1998a, Steinacker et al. 2000, 2004; Meeusen et al. 2004). The results of the research devoted to this subject is far from unanimous, mostly because of pre-analytical factors, i.e., factors that occur prior to the final analysis (time of sampling, food intake, time after the end of exercise, gender, age...) may influence the hormonal profile. In addition, measuring methods and/or detection limits of the analytical equipment used may differ between studies. Testing of central hypothalamic/pituitary regulation requires functional tests which are considered invasive and require diagnostic experience, and these tests are time consuming and expensive. Finally, the distinguishing characteristic of endocrine systems is the feedback control of hormone production. Virtually all hormones are under feedback control, some by the peripheral hormones themselves, some by other hormones or cytokines, peripheral metabolites, osmolality, etc. This feedback relationship is the reason why simultaneous assessment of hormone/effector pairs is frequently necessary for the assessment of hormonal status, taking also into consideration the fact that physiological processes related to endocrine regulation are influenced by more than a single hormone in a multi-level integrated way (Duclos 2008).

For a long time the resting plasma testosterone/cortisol ratio was considered as an indicator of the overtrained state. This ratio decreases in relation to the intensity and duration of training and it is evident that this ratio indicates only the actual physiological strain of training and cannot be used for diagnosis of OR or the OTS (Lehmann et al. 1998, 2001; Urhausen et al. 1995; Duclos 2008).

Most of the literature agrees that OR and the OTS must be viewed on a continuum with a disturbance, an adaptation, and finally a maladaptation of the hypothalamic pituitary adrenal axis (HPA) and all other hypothalamic axes (Lehmann et al. 1993b, 2001; Meeusen 1998; Meeusen et al. 2004, Urhausen et al. 1995, 1998b). For example, the HPA adaptation to normal training is characterised by increased $\mathrm{ACTH} /$ cortisol ratio only during exercise recovery (due to decreased pituitary sensitivity to cortisol) (Lehmann et al. 1993b; Duclos et al. 1997, 1998), and by modulation 
of tissue sensitivity to glucocorticoids (Duclos et al. 1999, 2003). However, it should be emphasized that during a resting day, in endurance-trained athletes $24 \mathrm{~h}$ cortisol secretion under non-exercising conditions is normal (Lancaster et al. 2004; Duclos et al. 1999, 2003). Accordingly, morning plasma cortisol concentration and $24 \mathrm{~h}$ urinary free cortisol (UFC) excretion in resting endurance-trained men are similar to those of age-matched sedentary subjects (Kern et al. 1995; Duclos et al. 1997; Gouarne et al. 2005). Since UFC represents an integrated measure of the $24 \mathrm{~h}$ cortisol secretion, this is in accordance with the previously reported normal diurnal HPA axis rhythm in endurance-trained men (Duclos et al. 1997, 2007). Finally, endurance-trained men maintain the seasonal rhythmicity of cortisol excretion; as in sedentary men the highest concentrations of urinary cortisol, morning plasma cortisol and saliva cortisol are observed during autumn and winter compared with spring and summer (Gouarne et al. 2005). Therefore, it can be concluded that resting cortisol is not a useful measurement.

There is no consensus with regard to plasma, $24 \mathrm{~h}$, or overnight urinary excretion of catecholamines, for monitoring the impact of the training load and/or an overload. Some studies report an increase, a decrease or no change of urinary catecholamine excretion (for a review see Duclos 2008) with successful training, OR or the OTS. Factors other than training load influence secretion and could result in variations between studies; these factors include: sampling methods, diurnal and seasonal variations of catecholamine excretion, sex difference effects. As the relationship between $24 \mathrm{~h}$ or nocturnal catecholamine urinary excretion and performance or training monitoring is inconclusive, it is thus inappropriate to use changes in catecholamine excretion as a tool to monitor training status. In the OTS, a decreased rise in pituitary hormones (ACTH, growth hormone, GH, luteinising hormone, $\mathrm{LH}$ and follicle stimulating hormone, FSH) in response to a stressful stimulus is reported (Barron et al. 1985; Lehmann et al., 1993b; Urhausen et al. 1995, 1998a; Wittert et al. 1996). But behind the seemingly uniform acute hormonal response to exercise, explaining the disturbance to the neuroendocrine system caused by the OTS is not that simple. Whether peripheral metabolic hormones can be used for OR/OTS diagnosis is currently under discussion.

A nutrient-sensing signal of adipose tissue is represented by leptin (Simsch et al. 2002) which like the glucoregulatory hormone insulin, interleukin-6 (IL-6) and the metabolic growth factor insulin-like growth-factor I (IGF-I) has been shown to decrease with training-induced catabolism like in OR. These signalling molecules have profound effects on the hypothalamus and are involved in the metabolic hormonal regulation of exercise and training (Steinacker et al. 2004). However, the same molecules respond to chronic energy deficiency which can be associated with endurance training and/or aesthetic sports (e.g., gymnastics), regardless of the training status (absence or presence of OR/OTS). Chronic energy deficiency (mainly glycogen depletion) certainly amplifies the stress hormone and cytokine responses to exercise and might also be one of the "triggering" factors that can lead to the induction of the OTS.

In addition to the need to study different hormonal axes in parallel, it is also important to consider the dynamics of hormonal responses. Indeed, the hormonal responses during exercise influence the hormonal responses during exercise recovery (Kanaley et al. 2001; Duclos et al. 2007; De GraafRoelfsema et al. 2007) and it is therefore important to study both phases of exercise. For this reason, a multiple-exercise test which gives the opportunity to measure the recovery capacity of the athlete 
but can also assess the ability to normally perform the second bout of exercise, could be useful to detect signs of the OTS and distinguish them from normal training responses or Functional OR.

Meeusen et al. (2004) published a test protocol with two consecutive maximal exercise tests separated by $4 \mathrm{~h}$. The use of 2 bouts of incremental exercise to volitional exhaustion to study neuroendocrine variations showed an exercise-induced increase of $A C T H$, prolactin (PRL) and GH to a two exercise bout (Meeusen et al. 2004). In normal healthy subjects the test reveals an increase in the circulating concentrations of the hormones after both the first and the second exercise bout. The test could be used as an indirect measure of hypothalamic-pituitary reactivity. Depending on the 'training' status of the athlete hormonal output after the second exercise test will be different. This test has the ability to distinguish a state of NFO from the OTS. In a Functional OR stage a less pronounced neuroendocrine response to a second bout of exercise on the same day is found, (Meeusen et al. 2004), while in a Non-Functional OR stage the hormonal response to a two bout exercise protocol shows a markedly higher elevation after the second exercise trigger (Meeusen et al. 2004). With the same protocol it has been shown that athletes suffering from the OTS have an extremely large increase in circulating hormone concentration after the first exercise bout, followed by a complete suppression in the second exercise bout (Meeusen et al. 2004, 2010). This could indicate a hypersensitivity of the pituitary followed by an insensitivity or exhaustion afterwards. Previous reports that used a single exercise protocol found similar effects (Meeusen et al. 2004). In a follow-up study they could clearly distinguish between NFO \& OTS athletes (Meeusen et al. 2010). It appears that the use of two exercise bouts is more useful in detecting OR for preventing overtraining. Early detection of OR may be very important in the prevention of OTS.

Other hormones such as leptin, adiponectin and ghrelin, as well as cytokines such as IL-6 and tumour necrosis factor-alpha have been recently investigated as possibilities for the monitoring of training (Jürimäe et al. 2011). The authors concluded that although some of these parameters measured in the fasting state or post-exercise may provide information about energetic regulatory mechanisms and may change after heavy training or inadequate recovery, there are no studies supporting the possible suitability of these variables as markers of training stress or for the prevention or diagnosis of OR or the OTS.

In conclusion, the endocrine system is one of the major systems involved in the responses to acute stress and adaptation to chronic stress. A great diversity of mechanisms is involved in such adaptation, acting at potentially all levels in the cascade leading to the biological effects of the hormones. However, the current information regarding the endocrine system and OR/OTS show that basal (resting) hormone measurements cannot distinguish between athletes who successfully adapt to OR and those who fail to adapt and develop symptoms of the OTS. Further studies using multiple exercise tests and/or multiple hormone analyses will be necessary for evaluating the possibility of a hormonal diagnostic test for OR/OTS.

\section{Problems with hormonal data:}

- Many factors affect blood hormone concentrations and these include factors linked to sampling conditions and/or conservation of the sampling: stress of the sampling, intra- and inter-assay coefficient of variability, 
- Food intake (nutrient composition and/or pre- versus post- meal sampling) can modify significantly either the basal concentration of some hormones (cortisol, DHEA-S, total testosterone) or their concentration change in response to exercise (cortisol, $\mathrm{GH}$ )

- Pulsatility of the secretion of some hormones which modulates the tissue sensitivity to these hormones

- In female athletes the hormonal response will depend on the phase of the menstrual cycle

- Aerobic and resistance protocols typically elicit different endocrine responses

- Hormone concentrations at rest and following stimulation (exercise = acute stimulus) respond differently

- Diurnal and seasonal variations of the hormones

- Stress-induced measures (exercise, pro-hormones, etc) need to be compared with baseline measures from the same individual

- Poor reproducibility and feasibility of some techniques used to measure some hormones (for example free testosterone by RIA instead of the reference method - reserved to some highly specialised centres - equilibrium dialysis)

- Hormonal responses to exercise can be prolonged during the recovery phase of exercise.

\section{PERFORMANCE TESTING}

In athletes who have been diagnosed as having the OTS, several signs and symptoms have been associated with this imbalance between training and recovery. However, reliable diagnostic markers for distinguishing between well-trained, OR and athletes having the OTS are lacking. A hallmark feature of the OTS is the inability to sustain intense exercise, a decreased sports-specific performance capacity when the training load is maintained or even increased (Urhausen et al. 1995, Meeusen et al. 2004). Athletes suffering from the OTS are usually able to start a normal training sequence or a race at their normal training pace but are not able to complete the training load they are given, or race as usual. The key indicator of the OTS can be considered an unexplainable decrease in performance. Therefore, an exercise / performance test is considered to be essential for the diagnosis of the OTS (Budgett et al. 2000; Urhausen et al. 1995).

It appears that both the type of performance test employed and the intensity/duration of the test are important in determining the changes in performance associated with the OTS. Debate exists as to which performance test is the most appropriate when attempting to diagnose OR and the OTS. In general, time to fatigue tests will most likely show greater changes in exercise capacity as a result of OR and the OTS than incremental exercise tests (Urhausen et al. 1998b, Halson \& Jeukendrup 2004). Time-trials reflect more accurately the sport specific task of most sports but have only rarely been used to objectively quantify the performance loss in OR (Halson et al. 2003). Additionally these tests allow the assessment of substrate kinetics, hormonal responses, and submaximal measures can be made at a fixed intensity and duration. In order to detect subtle performance decrements it might be better to use sports specific performance tests. Tests of high-intensity exercise performance may be appropriate in some sports. For example, isokinetic strength and power were shown to be decreased in 7 overreached rugby players (Coutts et al. 2007b), but increased after one week of taper. Problems with performance testing

- Baseline measures are often not available and therefore, the degree of performance limitation may not be exactly determined. Individual comparative values are mandatory 
- The intensity and reproducibility of the test should be sufficient to detect differences (max test; time trial)

- Necessity of highly standardised conditions from one test to another and from one laboratory to another

- Many performance tests are not sport-specific

- Submaximal ergometric test results do not seem to produce significant results (Urhausen et al. 1998a), but repeated maximal tests, required for assessment of an individual baseline measure, are difficult to obtain in athletes

- In this regard, since adequate standardization of laboratory tests is problematic, it may be that index training sessions recorded by coaches are better candidates to demonstrate the magnitude, timing and pattern of performance decrements

\section{PSYCHOLOGY}

The presence of psychological symptoms in cases of OTS has long been acknowledged (Darling, 1901) but systematic study on this topic did not begin until William Morgan's research in the 1980s on college swimmers and athletes in other sports. Using the Profile of Mood States (POMS) (Morgan et al. 1987a), a questionnaire that measures both general and specific moods, athletes were found to consistently report elevations in negative moods (tension, depression, anger, fatigue, confusion) and decreases in the positive mood of vigour during periods of rigorous training. More frequent assessments indicated mood state exhibits a predictable dose-response relationship with training whereby disturbances increase in a step-wise fashion as training loads rise in volume or intensity, with the peak of training and mood disturbance coinciding. Conversely, training tapers usually result in a reduction in negative moods and an increase in vigour such that at the end of a taper the mood scores return to the positive pattern typically observed at the outset of the season, referred to as the iceberg profile (Morgan et al. 1987a,b; Raglin et al. 1991). Dose-response relationships between training load and mood state have since been observed in studies involving more than 1,000 athletes in a variety of endurance and non-endurance sports requiring rigorous training regimens (Raglin \& Wilson, 2000). Research also indicates mood responses of men and women athletes do not differ except when they are exposed to significantly different training regimens (Morgan et al. 1987b; Raglin et al. 1990). Similar dose-response patterns have also been observed using simple self-report measures of muscle soreness, appetite, sleep disturbances, "heaviness" and perception of effort (Kenttä et al. 2001 Morgan et al. 1988a; O'Connor et al. 1997; Raglin \& Wilson 2000), indicating perceptual responses to increased training are global and systemic in nature, although the magnitude of change differs across measures (Morgan et al. 1988b; Raglin \& Wilson 2000).

When conditioning programmes involve rapid increases in training load over a course of days the instructions to complete psychological measures should, if possible, be modified to yield a more transient, state measure of mood by having subjects respond according to how they feel "today" or "right now". Research reveals as few as two days of intensified training can result in significant increases in POMS measures (O'Connor 1997) and scores on other psychological scales which precede changes in commonly used biochemical markers of training stress such as cortisol (Coutts et al. 2007a; O'Connor et al. 1989). More important for the standpoint of monitoring, athletes with signs of OTS typically exhibit both a greater increase in total mood disturbance and a different pattern of mood disturbance compared with athletes undergoing the same training who remain free from symptoms (Raglin \& Morgan, 1994). Specifically, among healthy athletes POMS fatigue and 
vigour show the largest shifts during peak overload training and depression increases the least of all POMS factors, whereas in athletes showing signs of the OTS depression increases the most of all POMS variables, with some reports (Morgan et al. 1987b) indicating up to $80 \%$ of affected athletes show signs of clinical depression.

\section{$\rightarrow$ Figure 2 : mood changes between healthy and overtrained athletes}

The previous findings have led to tests of mood state monitoring as a means to modulate training load with the goal of reducing the incidence of OTS. This intervention paradigm involved reducing the training load of athletes possessing excessively elevated POMS total mood total mood disturbance scores until scores fell within an acceptable range established a priori using either offseason baseline of each athlete (Berglund \& Safstrom, 1994), or the mean value for teammates undergoing the same training regimen (Raglin, 1993). Conversely, training loads were increased in athletes exhibiting only minor mood disturbances, and this intervention was nearly as frequent as cases in which training loads were reduced (Berglund \& Safstrom, 1994). Both studies reported a reduced incidence of OTS compared with previous rates, but replications incorporating involving larger samples and adequate control conditions remain needed.

Although research generally supports the use of psychological assessments for identifying individuals at risk of developing OTS, several potential problems exist that can constrain accuracy. The most serious among these is response distortion, wherein subjects falsely complete psychological questionnaires, particularly those with items of a sensitive or personal nature. The most common form of response distortion involves social desirability or "faking good" in which individuals answer items in order to present themselves in a uniformly positive light. Factors that can increase the likelihood of response distortion include coercion, the demand characteristics associated with the experimental hypothesis, or in the case of overtraining studies, "faking bad" in order to have one's training load reduced. Administering questionnaires repeatedly over an extended period of time can sometimes result in a form of response distortion in which participants respond to questions in a random manner. The risk of response distortion can be reduced by including research team members who are trained in the proper administration of psychological questionnaires, providing athletes clear and guaranteed assurances their data will remain confidential and not be used for selection purposes, and by carefully explaining the rationale of using psychological assessments while emphasizing there are no right or wrong ways to respond to the questionnaires.

A separate concern regarding the POMS is the finding that the sensitivity of the mood subscales to training load is not uniform. Some factors, particularly confusion barely change even following large increases in training load in either healthy or overtrained athletes, whereas other POMS subscales are responsive to non-training related sport stressors (Raglin et al. 1991). For example, POMS tension scores often remain elevated or even increase during training tapers, most likely because this factor is particularly sensitive to the impending stress of major competitions (Raglin et al. 1991). At a more fundamental level the POMS was designed for use in general circumstances and samples, and many sport psychologists contend sport-specific questionnaires should provide greater sensitivity and specificity for assessing athletes in the unique environment of sport. Consequently, several hundred sport specific psychological measures of personality, motivation and mood have been developed, including several for NFOR/OTS. In the case of OTS the decision to employ a general or 
sport-specific measure depends not only on published evidence of its predictive efficacy and construct validity, but also the theoretical orientation of the researcher. If it is believed the risk of OTS is a function of the sum total of stressors an athlete is exposed to-be they training related or not-then a non-specific questionnaire which captures broad moods, feelings or perceptions would be most appropriate. Conversely, if non-sport related stressors (e.g., psychosocial stressors, time zone travel) are viewed as inconsequential or only minor contributors to the OTS, then questionnaires delimited to items particular to the context of training should be employed.

For these and other reasons researchers have developed POMS based overtraining scales in the attempt to enhance its sensitivity. Raglin and Morgan (1994) created a Training Distress Scale (TDS) based on discriminant function analyses of POMS data from 186 healthy and overtrained college swimmers. A TDS spreadsheet may be accessed at http://champ.usuhs.mil/choptimize.html. The 7item (five depression, two anger) TDS was more accurate in identifying overtraining athletes compared with predictions using POMS total mood disturbance scores or depression scores, and subsequent research (Raglin \& Wilson 2000; Kenttä et al. 2001) using translations of the scale in several languages found TDS scores to be elevated in young swimmers reporting OTS. Kenttä et al. (2006) created a POMS energy index measure by subtracting fatigue from vigour scores to study 11 elite kayakers during an intensive 3-week training camp. The researchers had athletes complete the entire POMS following practice each day and in the morning before practice to assess mood state following training and recovery. POMS energy index scores were responsive to both training stress and recovery whereas depression scores were unchanged, suggesting to the authors the index could be a useful tool to reduce NFOR during intense but brief training cycles.

Several sport specific OTS scales have been developed using theoretical assumptions about what psychological and behavior factors should be associated with the OTS. Among them the most extensively studied has been the Recovery-Stress Questionnaire for Athletes (RESTQ-Sport) (Kellmann \& Kallus, 2001), a 77-item questionnaire encompassing 19 separate factors that assess both overtraining and recovery responses in endurance athletes. Monitoring the current levels both of stress and recovery has the possible advantage that problems may be detected before symptoms of overtraining and staleness (e.g., drowsiness, apathy, fatigue, irritability) are likely to appear. However, stress and recovery are often different in their time course. Although concerns with its factor structure have been expressed by other researchers (e.g., Davis et al. 2007), research indicates the RESTQ is responsive to changes in training load, particularly in athletes with signs of OTS (Kellmann \& Günther, 2000). Other less well-documented OTS scales include the SFMS, a 54-item forced-choice (i.e., yes-no) questionnaire that assesses whether athletes have experienced mood disturbances and various symptoms of overtraining during the previous month (cited in Elloumi et al. 2004) and the Daily Analyses of Life-Demands in Athletes (DALDA), 50-item scale with two sections assessing general and sport related stresses (Rushall, 1990) experienced over the past day using a three point Likert format.

In summary, research has provided general support for the efficacy of psychological assessments in both basic and applied research involving athletes undergoing overload training. There remains, however, a need for systematic study of the relative efficacy (i.e., sensitivity and specificity) of promising measures, and tests to establish protocols that effectively integrate psychological information with biological assessments to enhance their efficacy. 


\section{Psychomotor Speed tests}

A relatively new but promising tool in the early detection of NFO and therefore a potential preventive tool in developing an OTS is the measurement of psychomotor speed. The advantage of psychomotor speed testing above most other tests, lays in the fact that it is easy to use in the (sport) field just by using a simple personal computer. The tests are non- invasive, resistant to conscious manipulation by the athlete and inexpensive.

It is well described that symptoms such as concentration and memory problems and cognitive complaints are common in patients suffering from chronic fatigue syndrome (Fry et al. 1991), symptoms also found in people suffering an OTS (Lehmann et al. 1993b, Shepard 2001). These similarities have led to the use of attention and reaction time tests for early detection of NFO and preventing OTS. Rietjens et al. (2005) used a reaction time test (Finger Pre-Cuing test) as a detection tool for NFO. They found a significant decrease in reaction time in a group of seven cyclists after they had doubled their training volume over a period of three weeks (Rietjens et al. 2005). Especially on the more difficult conditions in the finger-precueing reaction time task, with the more easy conditions being insensitive to OR. This outcome suggests that task complexity is an important mediating variable in the relationship between $O R$ and brain functioning. In line with these findings Nederhof et al. (2006) described a decrease in reaction time in five NFO cyclists after a two-week training camp. In a later follow up study Nederhof and colleagues confirmed these findings (Nederhof et al. 2007, 2008).

More recently Hynynen et al. (2008) presented data in which OTS cyclists scored a significantly higher number of mistakes during a STROOP test. All these studies strongly suggest that central fatigue is an early (and maybe the most early) manifestation of OR. This suggestion is ratified by the findings of Tergau et al. (2000) who found an intra-cortical facilitation increase after exercise, indicating motor cortex fatigue.

These findings indicate that reaction and attention tests are promising tools in early detection of NFO and preventing the OTS. However more scientific studies are needed to find out which kind of psychomotor speed tests are the most sensitive for detecting NFO/OTS.

\section{Potential problems with psychological assessments}

- Mood state and other factors can be influenced by stressors unrelated to training and recovery

- It remains unclear if intervention paradigms based on psychological information should employ off-season baseline mood scores (i.e., intra-individual criterion), team averages (i.e., inter-individual) or combinations of baseline and training values would be more effective

- Psychological measures can be biased or rendered invalid by various forms of faking (e.g., social desirability), or overuse.

- Psychological tests must be administered with the appropriate instructional set (e.g., "right now", "today", "last week including today.") based on the training paradigm. Care must be taken with state (i.e., "right now") measures of mood as they can be influenced by extraneous factors.

- Care needs to be taken to explain the potential value of psychological measures to coaches and athletes who may be reluctant or sceptical. Researchers should be trained in the administration and interpretation of the measures employed. 


\section{PHYSIOLOGY}

There have been several proposals as to which physiological measures might be indicative of OR or the OTS. Reduced maximal heart rates after increased training may be the result of reduced sympathetic nervous system activity, of a decreased tissue responsiveness to catecholamines, of changes in adrenergic receptor activity, or may simply be the result of a reduced power output achieved with maximal effort. Several other reductions in maximal physiological measures (oxygen uptake, heart rate, blood lactate) might be a consequence of a reduction in exercise time and not related to abnormalities per sé, and it should be noted that changes of resting heart rate are not consistently found in athletes suffering from the OTS (Urhausen \& Kindermann 2002).

Heart rate variability (HRV) analysis has been used as a measure of cardiac autonomic balance, with an increase in HRV indicating an increase in vagal (parasympathetic) tone relative to sympathetic activity (Uusitalo et al. 2000). Numerous studies have examined the effects of training on indices of HRV, but to date few studies have investigated HRV in overreached or OTS athletes, with studies showing either no change (Achten \& Jeukendrup 2003; Hedelin et al. 2000a; Uusitalo et al. 1998), inconsistent changes (Uusitalo et al. 2000) or changes in parasympathetic modulation (Hedelin et al. 2000b).

Hedelin et al. (2000a) increased the training load of 9 canoeists by 50\% over a 6-day training camp. Running time to fatigue, $\mathrm{VO}_{2} \max$, submaximal and maximal heart rates and maximal blood lactate production all decreased in response to the intensified training; however, all indices of HRV remained unchanged. On average, there were no significant changes in low frequency power, high frequency power, total power or the ratio of low to high frequency power, both in the supine position and after head-up tilt. Similarly, Uusitalo et al. (1998) reported no change in intrinsic heart rate and autonomic balance in female athletes following 6-9 weeks of intensified training. This involved the investigation of autonomic balance assessed by pharmacological vagal and B-blockade. In addition, both the time domain and power spectral analysis in the frequency domain were calculated during rest and in response to head-up tilt. Results suggest that $\mathrm{HRV}$ in the upright position had a tendency to decrease in response to intensified training in the subjects who were identified as "overtrained" (Uusitalo et al. 2000). This may indicate vagal withdrawal and/or increased sympathetic activity. However, between-subject variability was high in this investigation. Finally, Hedelin et al. (2000a) reported increased HRV and decreased resting heart rate in a single "overtrained" athlete when compared to baseline measures. In comparison to normally responding subjects examined during the same period, the "overtrained" subject exhibited an increase in high frequency and total power in the supine position during intensified training, which decreased after recovery. The increase in high frequency power was suggested to be most likely the result of increased parasympathetic activity (Hedelin et al. 2000b).

Lamberts et al. (2010) proposed that the heart rate return (HRR) 1 min after high intensity interval exercise could serve to monitor training as it showed some correlation with the evolution of time trial performance after 4 weeks in 14 moderately well trained cyclists, but to date there are no published results available from athletes in OR or OTS. 
In a very recent study (Buchheit 2011) in young soccer players, a decrease of submaximal heart rate, a faster return of heart rate after exercise and an increase of vagal indices of HRV were associated with some positive adaptations to training, but the opposite was not true as "negative" changes of theses markers were not indicators of a performance decline.

A meta-analysis (Bosquet et al. 2008) concluded that short term ( $<2$ weeks) overload training results in an increased resting heart rate (mean value $+4.5 \mathrm{bpm})$, decreased maximal heart rate $(-7.5 \mathrm{bpm})$ and a higher ratio between low and high frequency HRV. However, this was no longer the case after longer intensified training interventions lasting $>2$ weeks, where the only significant difference remained a decreased maximal heart rate $(-3.6 \mathrm{bpm})$.

Concerning the assumption often claimed in a clinical context that cardiac complications such as arrhythmias or other ECG changes discovered in athletes could be explained by a state of OR or OTS, this hypothesis does not find any support by any study inducing OR or OTS. However, it should be mentioned that an infectious disease - maybe facilitated by the intermittently depressed immunological state - occurring in an athlete engaged in heavy training may expose the individual to a higher risk of cardiac complications including a higher heart rate, extrasystoles and even myocarditis (Friman and Wesslén 2000).

\section{Problems with physiological measures}

- HRV seems a tool in theory, but does not provide consistent results. One needs to be careful when using HRV as an outcome measure since there are many different ways to record and calculate the data. Currently there is no consensus regarding the required standardization and the method of measurement.

- The present data do not allow to distinguish between changes in physiological measures resulting from functional OR, non-functional OR and OTS

\section{IMMUNE SYSTEM}

There are many reports on URTI due to increased training, and also in OR and OTS athletes. It seems feasible that intensified training (leading to OR or the OTS) may increase both the duration of the socalled "open window' and the degree of the resultant immunodepression. However, the amount of scientific information to substantiate these arguments is limited. More data are available that each bout of prolonged and intensive exercise has transient but significant, wide ranging effects on the immune system (Gleeson, 2007; Nieman, 1997). Heavy exertion leads to alterations in immunity and host pathogen defence, and elevations in stress hormones, pro-and anti-inflammatory cytokines, and reactive oxygen species. The exercise-induced immune perturbations and physiologic stress are associated with an elevated risk of URTI, especially during the 1-2 week period following competitive marathon and ultramarathon race events (Nieman 2009). These data imply that chronic immune dysfunction and increased URTI symptomatology may result when exercise training is intensified leading to OR and OTS, but few well-designed studies have been conducted to verify this hypothesis.

Several studies that have investigated the effects of short periods (typically 1-3 weeks) of intensified training on resting immune function and on immunoendocrine responses to endurance exercise indicate that several indices of neutrophil function appear to be sensitive to the training load. A 2week period of intensified training in well-trained triathletes was associated with a $20 \%$ fall in the 
bacterially-stimulated neutrophil degranulation response (Robson et al. 1999). In another study, neutrophil and monocyte oxidative burst activity, mitogen-stimulated lymphocyte proliferation and percentage and number of T-cells producing interferon- $\gamma$ were lower at rest following one week of intensified training in cyclists (Lancaster et al. 2004). Other leukocyte functions including Tlymphocyte $\mathrm{CD}^{+} / \mathrm{CD} 8^{+}$ratios, lymphocyte antibody synthesis and natural killer cell cytotoxic activity have been shown to be lower following increases in the training load in already well-trained athletes (Verde et al. 1992). Several studies have documented a fall in salivary IgA concentration with intensified training and some, though not all have observed a negative relationship between salivary IgA concentration and occurrence of URTI (Gleeson 2000; Fahlman and Engels 2005; Neville et al. 2008; Bishop \& Gleeson 2009; Gleeson et al. 2011). Thus, with sustained periods of heavy training, several aspects of both innate and adaptive immunity are depressed. Low levels of salivary IgA concentration or secretion rate and high anti-inflammatory cytokine responses to antigen challenge may predispose to high respiratory illness susceptibility in athletes (Gleeson 2000; Fahlman \& Engels 2005; Gleeson et al. 2011). Several studies have examined changes in immune function during intensive periods of military training (Carins \& Booth 2002; Tiollier et al. 2005; Castell et al. 2010). However, this often involves not only strenuous physical activity, but also dietary energy deficiency, sleep deprivation and psychological challenges. These multiple stressors are likely to induce a pattern of immunoendocrine responses that amplify the exercise-induced alterations.

Studies that have examined athletes exposed to a long-term training periods (e.g., over the course of a 5-10 month competitive season) have shown a general trend of depression of both systemic and mucosal immunity (Baj et al. 1994; Bury et al. 1998; Gleeson et al. 1995, 1999; Gleeson 2000; Gleeson 2005; Morgado et al. 2011). In these studies depressed immunity was most commonly observed either at the end of the season or following the most intensive periods of training and/or competition. Although elite athletes are not clinically immune deficient, it is possible that the combined effects of small changes in several immune parameters may compromise resistance to common minor illnesses such as URTI. Protracted immune depression linked with prolonged training may determine susceptibility to infection, particularly at times of major competitions. However, it might just be that the increased URTI incidence reflects the increased stress associated with increased training, regardless of the response of the athlete to the increased physical stress. Furthermore, symptoms of respiratory illness reported by some athletes may be due to airway inflammation from non-infectious causes (Bermon 2007; Cox et al. 2008; Walsh et al. 2011) rather than actual infection with a pathogen.

Whether immune function is seriously impaired in athletes suffering from the OTS is unknown due to insufficient scientific data. However, anecdotal reports from athletes and coaches of an increased infection rate with OTS (Smith 2000) have been supported by a few empirical studies (Kingsbury et al. 1998; Reid et al. 2004). In a cohort study of highly trained athletes prior to the Olympic Games, over $50 \%$ of the athletes who reported symptoms of "overtraining" presented with infection compared with none of the athletes in the overreached group (Kingsbury et al. 1998). In junior rowers, studied during and following a training camp (functional OR), $40 \%$ of the male subjects had URTI (Steinacker et al. 2002). In a study by Reid et al. (2004) 41 competitive athletes with persistent fatigue and impaired performance had a thorough medical examination which identified medical conditions with the potential to cause fatigue and/or recurrent infections in $68 \%$ of the athletes. The most common 
conditions were humoral immune deficiency and unresolved viral infections. Evidence of Epstein-Barr virus reactivation was detected in $22 \%$ of the athletes tested. Adventure racing over a 4-5 day period has been linked to significant mood state disruption and elevated URTI rates (Anglem et al., 2008). Thus, it seems plausible that a significant number of athletes who are diagnosed as suffering from the OTS may experience increased URTI.

There are only a few reports of differences in immune function status in "overtrained" athletes compared with healthy trained athletes (e.g., Mackinnon \& Hooper 1994; Gabriel et al. 1998) and most studies on "overtrained" athletes have failed to find any differences (Rowbottom et al. 1995; Mackinnon et al. 1997). Circulating numbers of lymphocyte subsets change with exercise and training. With heavy training, the T-lymphocyte $\mathrm{CD}^{+} / \mathrm{CD}^{+}$(helper/ suppressor) ratio falls. However, this has not been shown to be different in athletes diagnosed as suffering from the OTS compared with healthy well-trained athletes. One study (Gabriel et al. 1998) has shown that the expression of other proteins on the cell surface of T-lymphocytes does seem to be sensitive enough to distinguish between the majority of "overtrained" athletes and healthy athletes. The expression of CD45RO on T-helper $\mathrm{CD}^{+}$cells (but not the circulating numbers of $\mathrm{CD} 45 \mathrm{RO}^{+} \mathrm{T}$-cells) was significantly higher in athletes suffering from the OTS compared with healthy well-trained controls. Using this indicator, "overtraining" could be classified with high specificity and sensitivity. However, CD45RO is a marker of T-memory cells and activated T cells. Thus, higher expression of CD45RO on T cells may merely be indicative of the presence of acute infection, which is, of course, a possible cause of the underperformance. Fry et al. (1994) reported a significant increase in activation markers (CD25, HLADR) in blood lymphocytes of "overtrained" athletes. Unresolved viral infections are not routinely assessed in elite athletes, but it may be worth investigating this in individuals experiencing fatigue and underperformance in training and competition. Thus, infection might be one of the 'triggering' factors that can lead to the induction of the OTS or in some cases the diagnosis of OTS cannot be differentiated from a state of post-viral fatigue such as that observed with episodes of glandular fever. In the OTS-diagnostic flowchart (figure 3) it is recommended, to evaluate for "primary" viral and bacterial infections and systemic inflammatory diseases before proceeding with the diagnostic workup in direction OTS. It is acknowledged in the flowchart, that secondary in the time course of OTS a reactivation of Epstein-Barr virus can be detected (Reid et al. 2004) which may contribute to the severity of symptoms. However, despite this distinction between "primary" and "secondary" infection may be in some cases clinically difficult, it may help in explanation and treatment of fatigue and underperformance related diseases.

In conclusion, it is clear that the immune system is sensitive to stress - both physiological and psychological - and thus, potentially, immune variables could be used as an index of stress in relation to exercise training. The current information regarding the immune system and overreaching confirms that periods of intensified training result in depressed immune cell functions with little or no alteration in circulating cell numbers. However, although immune parameters change in response to increased training load, these changes do not distinguish between those athletes who successfully adapt to overreaching and those who maladapt and develop symptoms of the OTS. Furthermore, at present it seems that measures of immune function cannot really distinguish OTS from infection or post-viral fatigue states. 


\section{Problems with immunological testing}

- Timing of the test (time of the day; time since last exercise session)

- Lack of consistency of the data in literature

- Time consuming and very expensive (for functional measures)

\section{Resistance Exercise}

Although most research on overtraining and overreaching has focused on endurance activities, some research has shed light on stressful training when using heavy resistance exercise, and is summarized in several reviews (Fry \& Kraemer 1977; Fry 1999; Fry et al. 2001, 2005). What has become clear is that excessively high volumes or intensities of resistance exercise can present considerably different physiological and performance profiles when compared to OT/OR with endurance activities. When excessive volumes of maximal loads are used for training, maximal muscular strength is one of the last performance measures to be adversely affected. On the other hand high speed (e.g., sprinting) and power appear to be more sensitive to the stressful resistance exercise training and are the first types of performance to decrease. Although not greatly studied, some data also indicates that psychological variables may be sensitive to resistance exercise OT/OR. From an endocrine perspective, although testosterone concentrations and the testosterone/cortisol ratio may decrease due to resistance exercise OT/OR, these cannot be used to define the presence of an OTS. Rather, these hormonal measures simply indicate the presence of stressful training. When resistance trained athletes are exposed to a repeated stressful training phase, the decreased hormonal response is lessened, suggesting that repeated training of this type may permit long-term training tolerance. The presence of an elevated acute sympathetic response with excessive resistance exercise loads supports the concept of a sympathetic overtraining syndrome. This in turn may contribute to downregulation of $\beta_{2}$ adrenergic receptors in the affected skeletal muscle (Fry et al. 2006). From a practical standpoint, the actual training programme must be carefully monitored to incorporate adequate recovery phases as needed. Finally, it is readily apparent that sport-specific training in addition to the resistance exercise programme can add to the training stresses and contribute to OT/OR (Moore et al. 2007).

Problems with Resistance Exercise OT/OR Research

- There are few research studies on resistance exercise OT/OR

- There are many variations of resistance exercise that make it difficult to study

- Muscular strength is usually preserved with resistance exercise OT/OR

- Delayed onset muscular soreness and muscle damage are not necessarily the same as resistance exercise OT/OR

- Few studies have monitored an adequate recovery period

Prevention

One general confounding factor when reviewing literature on OTS is that the definition and diagnosis of OR and the OTS is not standardised. One can even question if in most of the studies subjects were suffering from OTS. Because the OTS is difficult to diagnose, authors agree that it is important to prevent OTS (Foster et al. 1988; Kuipers 1996; Uusitalo 2001). Moreover, because the OTS is mainly due to an imbalance in the training recovery ratio (too much training and competitions and too little recovery), it is of utmost importance that athletes record daily their training load, using a daily training diary or training log (Foster et al. 1996, 1988; Foster 1998). The four methods, most frequently used to monitor training and prevent overtraining are: retrospective questionnaires, 
training diaries, physiological screening and the direct observational method (Hopkins 1991). Also the psychological screening of athletes (Berglund \& Safstrom 1994; Hooper et al. 1995; Hooper \& McKinnon 1995; McKenzie 1999; Raglin et al. 1991; Urhausen et al. 1998b; Morgan et al. 1988) and the Ratings of Perceived Exertion (RPE) (Acevedo et al. 1994; Callister et al. 1990; Foster et al. 1996; Foster 1998; Hooper et al. 1995; Hooper \& McKinnon 1995; Kentta \& Hassmen 1998; Snyder et al. 1993) have received more and more attention nowadays.

Hooper et al. (1995) used daily training logs during an entire season in swimmers to detect staleness (OTS). The distances swum, the dry-land work time and subjective self-assessment of training intensity were recorded. In addition to these training details the swimmers also recorded subjective ratings of quality of sleep, fatigue, stress and muscle soreness, body mass, early morning heart rate, occurrence of illness, menstruation and causes of stress. Swimmers were classified as having the OTS if their profile met five criteria. Three of these criteria were determined by items of the daily training logs: fatigue ratings in the logs of more than 5 (scale 1-7) lasting longer than 7 days, comments in the page provided in each log that the athlete was feeling that he/she responded poorly to training and a negative response to a question regarding presence of illness in the swimmer's log, together with normal blood leukocyte count.

Foster et al. $(1996,1998)$ have determined training load as the product of the subjective intensity of a training session using 'session RPE' and the total duration of the training session expressed in minutes. If these parameters are summated on a weekly basis it is called the total training load of an individual. The 'session RPE' has been shown to be related to the average percent heart rate reserve during an exercise session and to the percentage of a training session during which the heart rate is in blood lactate derived heart rate training zones. With this method of monitoring training they have demonstrated the utility of evaluating experimental alterations in training and have successfully related training load to its performance (Foster et al. 1996). Foster et al. (2001) have demonstrated that athletes often do not perform the same training load prescribed by coaches. In particular they noted that on days the coaches intended to be 'easy', athletes often performed meaningfully longer and/or more intense training. These data fit well with the concept that OTS is a failure of the workrecovery relationship, often in the direction of athletes failing to take appropriate recovery. However, training load is clearly not the only training related variable contributing to the genesis of OTS. So additionally to the weekly training load, daily mean training load as well as the standard deviation of training load were calculated during each week. The daily mean divided by the standard deviation was defined as the monotony. The product of the weekly training load and monotony was calculated as strain. The incidence of simple illness and injury was noted and plotted together with the indices of training load, monotony and strain. They noted the correspondence between spikes in the indices of training monotony and strain and subsequent illness or injury and thresholds that allowed for optimal explanation of illnesses were computed (Foster 1998). The data in this study (Foster 1998) was suggested by earlier data by Bruin et al. (1994) in race horses. The horses responded appropriately to progressive increases in the training load until the normal recovery days were made harder (e.g. the monotony of training was increased). At this point the running performance of the horses deteriorated and the horses demonstrated behavioural signs consistent with an equine version of OTS (e.g. being "off their feed", which included loss of appetite, biting their handlers and kicking their stalls). This finding of a sudden deterioration of performance with loss of 
normal regeneration is also consistent with the differences in training programme design by coaches versus execution by athletes (Foster et al 2001).

One of the disadvantages of the traditional 'paper \& pencil' method is that data collection can be complicated, and that immediate feedback is not always possible. Another problem is that when athletes are on an international training camp or competition, immediate 'data computing' is not possible. It might therefore be useful to have an 'on-line' training log which has specific features in detecting not only slight differences in training load, but also the subjective parameters (muscle soreness, mental and physical well-being) that have been proven to be important in the detection of the OTS.

Strategies to reduce the symptoms of OR and reduce the risk of developing OTS

Both in the earlier data, reviewed in the ECSS 2006 consensus statement, and in the more contemporary data in this document, there is virtually no evidence suggesting that OTS can be 'treated'. Like a massive orthopeadic injury, OTS (and even NFOR) is just as debilitating, and takes a substantial time for recovery to occur spontaneously. Rest and very light training seem to be the only therapeutic agents capable of effecting recovery. The overwhelming impression, particularly in the evidence that has emerged since 2006, is that the emphasis needs to be on prevention of NFOR and OTS (mostly by appropriate periodization of the training program with careful focus on including, and executing, appropriate recovery time into the training program) and on early diagnosis of NFOR and OTS, which at least in principle might shorten the recovery time.

Rest and sleep

One of the most obvious methods for managing fatigue and enhancing recovery is adequate passive rest and obtaining sufficient sleep. It is generally recommended that athletes should have at least one passive rest day each week, as the absence of a recovery day, especially during intensified training periods, is closely related to the onset of signs of OR and under-recovery (Bruin et al. 1994). A passive rest day can also act as a 'time-out' period for athletes and prevent them from becoming totally preoccupied with their sport and possibly encourage them to pursue a different (passive) interest. Such distractions from the daily routine of training may alleviate boredom and reduce stress perception.

Sleep is an essential part of fatigue management, as persistent sleep loss can negatively impact on the quality of a training session and general well-being. The primary need for sleep has been hypothesized as being neurally based rather than a requirement for restitution of other biological tissues (Horne \& Pettitt 1984). Therefore, with inadequate sleep, cognitive functions are likely to be impaired, especially the ability to concentrate. Individuals have different requirements for sleep and to prescribe the dose of sleep that a highly trained athlete requires would be erroneous. The general advice is to sleep for the amount of time that is required to feel wakeful during the day, which may vary considerably between individuals.

\section{Nutrition}

Because OR is brought about by high-intensity training with limited recovery, it is thought that the fatigue and underperformance associated with OR are at least partly attributable to a decrease in muscle glycogen levels. Decreased glycogen levels can result in disturbances of the endocrine milieu. Glycogen depletion results in higher circulating levels of catecholamines, cortisol, and glucagon in response to exercise while insulin levels are very low. Such hormonal responses will result in changes 
in substrate mobilization and utilization (for instance, high adrenaline levels in combination with low insulin will increase lipolysis and stimulate the mobilisation of fatty acids). Because repeated days of hard training and carbohydrate depletion seem to be linked to the development of OR, it is tempting to think that carbohydrate supplementation can reverse the symptoms (Snyder 1999). In a group of runners who ran 16 to $21 \mathrm{~km}$ on a daily basis for 7 days and treated all those runs as races, performance dropped significantly when a moderate carbohydrate intake of $5.5 \mathrm{~g} / \mathrm{kg}$ body mass/day was maintained (Achten et al. 2004). The runners also displayed a range of symptoms indicating that they were overreached. But when the daily carbohydrate was increased to $8.5 \mathrm{~g} / \mathrm{kg}$ body mass/day, the drops in performance were much smaller and OR symptoms were reduced. Recovery from this week of hard training was more complete with the high-carbohydrate treatment. In this study the dietary intake was strictly controlled and the subjects were fed to maintain energy balance. In a follow-up study subjects received a carbohydrate supplements before, during and after training sessions, but their dietary intake the rest of the day was recorded but not controlled (Halson et al. 2004). In this study a group of well-trained cyclists were required to perform 8 days of intensive endurance training (normal training volume was doubled). This training was performed on two occasions separated by a washout, or recovery, period of at least 2 weeks. On one occasion subjects consumed a $2 \%$ carbohydrate solution before, during, and after training (low-CHO), and on the other occasion subjects consumed a $6.4 \%$ carbohydrate solution before and during training and a $20 \%$ carbohydrate solution after training (high-CHO). Total carbohydrate intake was $6.4 \mathrm{~g} / \mathrm{kg}$ body mass/day with low-CHO and $9.4 \mathrm{~g} / \mathrm{kg}$ body mass/day with high- $\mathrm{CHO}$. The intensified training protocol induced OR as indicated by a decrease in performance (time to fatigue at $74 \%$ of aerobic capacity), although the decrease in performance was significantly less with high- $\mathrm{CHO}$, suggesting that high- $\mathrm{CHO}$ diets can reduce the severity of OR. Alteration of mood state (assessed by POMS questionnaire) and hormonal disturbances in the response to exercise were also less on high-CHO compared with low$\mathrm{CHO}$. By requiring the subjects to consume supplements that contained a large amount of carbohydrate, the total energy intake increased as well (13.0 versus $16.5 \mathrm{MJ} /$ day for low-CHO and high-CHO, respectively). Athletes in hard training seem to reduce (or not increase) their spontaneous food intake, and unless they supplement with carbohydrate they may be in negative energy balance during periods of intensified training. It also appeared that the amount of carbohydrate ingested during training influenced the length of time needed for recovery. After 2 weeks of recovery (reduced volume and intensity) from intensified training, performance remained below that of baseline for the low-CHO treatment, whereas performance improved compared with baseline after 2 weeks of recovery from intensified training with the high- $\mathrm{CHO}$ condition.

Besides carbohydrate depletion, dehydration and negative energy balance can increase the stress response (increased catecholamines, cortisol, and glucagon, while insulin levels are reduced), which increases the risk of developing OR symptoms. Thus, in order to reduce the symptoms of OR and reduce the risk of developing the OTS during periods of intensive training, athletes should be encouraged to increase their fluid, carbohydrate and energy intake to meet the increased demands. Additional carbohydrate should not be at the expense of reduced protein intake as there is some evidence that insufficient protein can also result in increased risk of OR (Kingsbury et al. 1988). Supplementation with amino acids (glutamine, branched chain amino acids), however, is not likely to reduce symptoms of fatigue and OR (Meeusen \& Watson 2007).

Considerations for coaches and physicians 
Until a definitive diagnostic tool for the OTS is present, coaches and physicians need to rely on performance decrements as verification that an OTS exists. However, if sophisticated laboratory techniques are not available, the following considerations may be useful :

- Maintain accurate records of performance during training and competition. Be willing to adjust daily training intensity/volume, or allow a day of complete rest, when performance declines, or the athlete complains of excessive fatigue

- Avoid excessive monotony of training

- Always individualise the intensity of training

- Encourage and regularly reinforce optimal nutrition, hydration status and sleep

- Be aware that multiple stressors such as sleep loss or sleep disturbance (e.g., jet lag), exposure to environmental stressors, occupational pressures, change of residence, and interpersonal or family difficulties may add to the stress of physical training

- Treat the OTS with rest! Reduced training may be sufficient for recovery in some cases of overreaching

- Resumption of training should be individualised on the basis of the signs and symptoms because there is no definitive indicator of recovery

- Communication with the athletes (maybe through an on-line training diary) about their physical, mental and emotional concerns is important

- Include regular psychological questionnaires to evaluate the emotional and psychological state of the athlete

- Maintain confidentiality regarding each athlete's condition (physical, clinical and mental).

- Importance of regular health checks performed by a multidisciplinary team (physician, nutritionist, psychologist...)

- Allow the athlete time to recover after illness/injury

- Note the occurrence of URTI and other infectious episodes; the athlete should be encouraged to suspend training or reduce the training intensity when suffering from an infection

- Always rule out an organic disease in cases of performance decrement

- Unresolved viral infections are not routinely assessed in elite athletes, but it may be worth investigating this in individuals experiencing fatigue and underperformance in training and competition

Moreover, when OTS is suspected, it is also of utmost importance to standardise the criteria used for diagnosis and/or, at least, as tools for the diagnosis of OTS are lacking, to standardise the criteria of exclusion of OTS (see figure 1 for the definition and table $1 \& 2$ ) .

\section{Conclusion}

A difficulty with recognising and conducting research into athletes with OTS is defining the point at which OTS develops. Many studies claim to have induced OTS but it is more likely that they have induced a state of OR in their subjects. Consequently, the majority of studies aimed at identifying markers of ensuing OTS are actually reporting markers of excessive exercise stress resulting in the acute condition of OR and not the chronic condition of OTS. The mechanism of the OTS could be difficult to examine in detail maybe because the stress caused by excessive training load, in 
combination with other stressors might trigger different "defence mechanisms" such as the immunological, neuroendocrine and other physiological systems that all interact and probably therefore cannot be pinpointed as the "sole" cause of the OTS. It might be that as in other syndromes (e.g., chronic fatigue syndrome, or burnout) the psychoneuroimmunology (study of brainbehaviour-immune interrelationships) might shed a light on the possible mechanisms of the OTS, but until there is no definite diagnostic tool, it is of utmost importance to standardise measures that are now thought to provide a good inventory of the training status of the athlete. A primary indicator of OR or OTS is a decrease in sport specific performance, and it is very important to emphasise the need to distinguish the OTS from OR and other potential causes of temporary underperformance such as anaemia, acute infection, muscle damage and insufficient carbohydrate intake.

The physical demands of intensified training are not the only elements in the development of the OTS. It seems that a complex set of psychological factors are important in the development of the OTS, including excessive expectations from a coach or family members, competitive stress, personality structure, social environment, relationships with family and friends, monotony in training, personal or emotional problems, and school- or work- related demands. While no single marker can be taken as an indicator of impending OTS, the regular monitoring of a combination of performance, physiological, biochemical, immunological and psychological variables would seem to be the best strategy to identify athletes who are failing to cope with the stress of training. We therefore propose a "Check List" that might help the physicians to decide on the diagnosis of OTS and to exclude other possible causes of underperformance (see table 2). 


\section{REFERENCES}

1. Acevedo E, Rinehardt K, Kraemer R. (1994) Perceived exertion and affect at varying intensities of running. Res Q Exerc Sport, 65(4), 372-376.

2. Achten J, Halson SL, Moseley L, Rayson MP, Casey AC, Jeukendrup AE. (2004) Higher dietary carbohydrate content during intensified running training results in better maintenance of performance and mood state. J Appl Physiol, 96, 1331-1340.

3. Achten J, Jeukendrup AE. (2003) Heart rate monitoring: applications and limitations. Sports Med , 33, 517-538.

4. Anglem N, Lucas S, Rose E, Cotter J. (2002) Mood, illness and injury responses and recovery with adventure racing. Wilderness Environ Med, 19, 30-38.

5. Baj Z, Kantorski J, Majewska E, Zeman K, Pokoca L, Fornalczyk E, Tchorzewski H, Sulowska Z, Lewicki R. (1994) Immunological status of competitive cyclists before and after the training season. Int J Sports Med., 15, 319-324.

6. Barron G, Noakes T, Levy W, Smidt C, Millar R. (1985) Hypothalamic dysfunction in overtrained athletes, J Clin Endocrin Metabol. , 60, 803-806.

7. Berglund B, Safstrom H, (1994) Psychological monitoring and modulation of training load of world-class canoeists. Med Sci Sports Exerc , 26, 1036-1040.

8. Bermon, S. (2007) Airway inflammation and upper respiratory tract infection in athletes: is there a link? Exerc Immunol Rev, 13, 6-14.

9. Bishop, N.C. \& Gleeson, M. (2009) Acute and chronic effects of exercise on markers of mucosal immunity. Front Biosci , 14, 4444-4456.

10. Bosquet L, Merkari S, Arvisais D, Aubert A. (2008) Is heart rate a convenient tool to monitor over-reaching? A systematic review of the literature. Br J Sports Med, 42, 709-714.

11. Bruin G, Kuipers $H$, Keizer $H$, Vandewalle H. (1994) Adaptation and overtraining in horses subjected to increasing training loads. J. Appl. Physiol. , 76, 1908-1913.

12. Bruin G, Kuipers H, Keizer HA, Vander Vusse GJ. (1994) Adaptation and overtraining in horses subjected to increasing training loads. J Appl Physiol , 76, 1908-1913.

13. Buchheit M, Simpson M, Al Haddad H, Bourdon P, Mendez-Villanueva A. (2012) Monitoring changes in physical performance with heart rate measures in young soccer players. Eur J Appl Physiol , 112(2), 711-723.

14. Budgett R, Newsholme E, Lehmann M, Sharp C, Jones D, Peto T, Collins D, Nerurkar R, White P. (2000) Redefining the overtraining syndrome as the unexplained underperformance0 syndrome. Br J Sports Med , 34, 67-68.

15. Bury T, Marechal R, Mahieu P, Pirnay F. (1998) Immunological status of competitive football players during the training season. Int J Sports Med , 19, 364-368.

16. Callister R, Callister R, Fleck S, Dudley G. (1990) Physiological and performance responses to overtraining in elite judo athletes. Med Sci Sports Exerc , 22(6), 816-24.

17. Carins J, Booth C. (2002) Salivary immunoglobulin-A as a marker of stress during strenuous physical training. Aviat Space Environ Med., 3, 1203-1207.

18. Castell L, Thake C, Ensign W. (2010) Biochemical markers of possible immunodepression in military training in harsh environments. Military Med, 175, 158-165.

19. Cizza G, Kvetnansky R, Tartaglia M, Blackman M, Chrousos G, Gold P. (1993) Immobolisation stress rapidly decreases hypothalamic corticotropin-releasing hormone secretion in vitro in the male 344/N fischer rat. Life Sci , 53, 233-240. 
20. Coutts A, Reaburn P, Piva T, Murphy A. (2007a) Changes in selected biochemical, muscular strength, power, and endurance measures during deliberate overreaching and tapering in rugby league players. Intern J Sports Med , 28, 116-124.

21. Coutts A, Wallace L, Slattery K. (2007b) Monitoring changes in performance, physiology, biochemistry, and psychology during overreaching and recovery in triathletes. Intern J Sports Med , 28, 125-134.

22. Cox A, Gleeson M, Pyne D, Callister R, Hopkins W, Fricker P. (2008) Clinical and laboratory evaluation of upper respiratory symptoms in elite athletes. Clin J Sport Med, 18, 438-445.

23. Darling E. (1901) The Medical Supervision of Athletes, Harvard Graduates Magazine , 10, 190196.

24. Davis H, Orzec T, Keelan P. (2007) Psychometric item evaluations of the Recovery-Stress Questionnaire for athletes. Psychol Sport Exerc, 8, 917-938.

25. De Graaf-Roelfsema E, Keizer H, Wijnberg I, van der Kolk J. (2007) Hormonal responses to acute exercise, training and overtraining. A review with emphasis on the horse. Vet.Q. , 29, 82-101.

26. Duclos M, Corcuff J-B, Arsac L, Moreau-Gaudry F, Rashedi M, Roger P, Tabarin A, Manier G. (1998) Corticotroph axis sensitivity after exercise in endurance-trained athletes. Clin Endocrinol, 8, 493-501.

27. Duclos M, Corcuff J-B, Rashedi M, Fougere V, Manier G. (1997) Trained versus untrained men: different immediate post-exercise responses of pituitary-adrenal axis. Eur J Appl Physiol , 75, 343-350.

28. Duclos M, Gouarne C, Bonnemaison D. (2003) Acute and chronic effects of exercise on tissue sensitivity to glucocorticoids. J Appl Physiol , 94, 869-875.

29. Duclos M, Guinot M, Le Bouc Y. (2007) Cortisol and GH: odd and controversial ideas. Appl Physiol Nutr Metab , 32, 895-903.

30. Duclos M, Minkhar M, Sarrieau A, Bonnemaison D, Manier G, Mormede P. (1999) Reversibility of endurance training-induced changes on glucocorticoid sensitivity of monocytes by an acute exercise. Clin Endocrinol , 1, 749-756.

31. Duclos M. (2008) A critical assessment of hormonal methods used in monitoring training status in athletes. Intern Sport Med Journal , 9 (2), 56-66.

32. Elloumi M, El Elj N, Zaouali M, Maso F, Filaire E, Tabka Z, Lac G. (2005) IGFBP-3, a sensitive marker of physical training and overtraining. Br J Sports Med, 39, 604-610.

33. Fahlman M, Engels H. (2005) Mucosal IgA and URTI in American college football players: a year longitudinal study. Med Sci Sport Exerc, 37, 374-380.

34. Folland J, Chong J, Copeman E, Jones D. (2001) Acute muscle damage as a stimulus for traininginduced gains in strength. Med Sci Sport Exer, 33, 1200-1205.

35. Foster C, Daines E, Hector L, Snyder A, Welsh R. (1996) Athletic performance in relation to training load. Wisconsin Med J, 95, 370-374.

36. Foster C, Heimann KM, Esten PL, Brice G, Porcari J. (2001) Differences in perceptions of training by coaches and athletes. SAfri I Sports Med , 8, 3-7.

37. Foster C, Snyder A, Thompson N and Kuettel K. (1998) Normalisation of the blood lactate Profile. Int J Sports Med, 9, 198-200.

38. Foster C. (1998) Monitoring training in athletes with reference to overtraining syndrome. Med Sci Sports Exerc, 30(7), 1164-8 .

39. Friman G, Wesslén L. (2000) Special feature for the Olympics: effects of exercise on the immune system: infections and exercise in high-performance athletes. Immun Cell Biol, 78, 510-522. 
40. Fry A, Hakkinen K, Kraemer W. (2001) Special considerations in strength training. In: W.J. Kraemer and K. Hakkinen (eds.) Handbook of Sports Medicine and Science - Strength Training for Sport, An IOC Medical Commission Publication, Blackwell Scientific, London, pp. 135-162.

41. Fry A, Kraemer W. (1997) Resistance exercise overtraining and overreaching. Sports Med, 23, 106-129.

42. Fry A, Kraemer W. (1997) Resistance exercise overtraining and overreaching: neuroendocrine responses, Sports Med, 23(2), 106-129.

43. Fry A, Schilling B, Weiss L, Chiu L. (2006) Beta ${ }_{2}$-adrenergic receptor down-regulation and performance decrements during high intensity overtraining. J Appl Physiol, 101, 1664-1672.

44. Fry A, Steinacker J, Meeusen R. (2005) Endocrinology of overtraining. In: W.J. Kraemer (ed.)The Encyclopedia of Sports Medicine. Blackwell Scientific, London, pp. 578-599.

45. Fry A.C. (1999) Overload and regeneration during resistance exercise. In: M. Lehmann, J.M. Steinacker, and U. Gastmann (eds.) Overload, Performance Incompetence, and Regeneration in Sport, Kluwer Academic/Plenum Plubishers, New York, pp. 149-162.

46. Fry R, Grove J, Morton A, Zeroni P, Gaudieri S, Keast D. (1994) Psychological and immunological correlates of acute overtraining. Br J Sports Med, 28, 241-246.

47. Fry R, Morton A, Keast D. (1991) Overtraining in athletes, Sports Med, 12, 32-65.

48. Gabriel H, Urhausen A, Valet G, Heidelbach U, Kindermann W. (1998) Overtraining and immune system: A prospective longitudinal study in endurance athletes. Med Sci Sports Exerc, 30, 11511157.

49. Gleeson M, Bishop N, Oliveira M, McCauley T, Tauler P, Muhamad A. (2012 - in press) Respiratory infection risk in athletes: association with antigen-stimulated IL-10 production and salivary IgA secretion. Scand J Med Sci Sports, DOI: 10.1111/j.1600-0838.2010.01272.x.

50. Gleeson M, McDonald W, Cripps W, Clancy L, Fricker P. (1995) The effect on immunity of longterm intensive training in elite swimmers. Clin Exp Immunol., 102, 210-216.

51. Gleeson M, McDonald W, Pyne D, Cripps A, Francis J, Fricker P, Clancy R. (1999) Salivary IgA levels and infection risk in elite swimmers. Med Sci Sports Exerc ,31, 67-73.

52. Gleeson M. (2000) Mucosal immune responses and risk of respiratory illness in elite athletes. Exerc Immunol Rev, 6: 5-42.

53. Gleeson M. (2004) Immune function and exercise. Eur J Sport Sci , 4(3), 52-66.

54. Gleeson M. (2007) Immune function in sport and exercise. J Appl Physiol, 103(2), 693-699.

55. Gouarne C, Groussard C, Gratas-Delamarche A, Delamarche P, Duclos M. (2005) Overnight urinary cortisol and cortisone add new insights into adaptation to training. Med Sci Sports Exerc, 37, 1157-1167.

56. Halson S, Bridge M, Meeusen R, Busschaert B, Gleeson M, Jones D, Jeukendrup A. (2002) Time course of performance changes and fatigue markers during intensified training in trained cyclists. J Appl Physiol, 93(3), 947-956.

57. Halson S, Jeukendrup A. (2004) Does Overtraining exist ? An analysis of overreaching and overtraining research. Sports Med, 34, 967-981.

58. Halson S, Lancaster G, Jeukendrup A, Gleeson M. (2003) Immunological responses to overreaching in cyclists. Med Sci Sports Exerc, 35, 854-861.

59. Halson S, Lancaster G, Achten J, Gleeson M, Jeukendrup A. (2004) Effect of carbohydrate supplementation on performance and carbohydrate oxidation following intensified cycling training. J Appl Physiol, 97, 1245-1253. 
60. Hedelin R, Kentta G, Wiklund U, Bjerle P, Henriksson-Larsen K. (2000a) Short-term overtraining: effects on performance, circulatory responses, and heart rate variability. Med Sci Sports Exerc, $32,1480-1484$.

61. Hedelin R, Wiklund U, Bjerle P, Henriksson-Larsen K. (2000b) Cardiac autonomic imbalance in an overtrained athlete. Med Sci Sports Exerc, 32, 1531-1533.

62. Hooper S, Mackinnon L. (1995) Monitoring overtraining in athletes. Recommendations. Sports Med, 20(5), 321-327.

63. Hooper SL, Mackinnon LT, Howard A, Gordon RD, Bachmann AW. (1995) Markers for monitoring overtraining and recovery. Med Sci Sports Exerc, 27(1), 106-112.

64. Hopkins W. (1991) Quantification of training in competitive sports. Methods and Applications. Sports Med, 12, 161-83.

65. Horne JA, Pettitt AN. (1984) Sleep deprivation and the physiological response to exercise under steady-state conditions in untrained subjects. Sleep, 7, 168-179.

66. Hynynen E, Uusitalo A, Konttinen N, Rusko H. (2008) Cardiac autonomic responses to standing up and cognitive task in overtrained athletes. Int J Sports Med, 29(7), 552-558.

67. Israel S. (1976) Zur Problematik des Übertrainings aus internistischer und leistungsphysiologischer Sicht. Medizin und Sport, 16(1), 1-12.

68. Jeukendrup A, Hesselink M, Snyder A, Kuipers H, Keizer H. (1992) Physiological changes in male competitive cyclists after two weeks of intensified training. Int J Sports Med , 13, 534-541.

69. Jürimäe J, Mäestu J, Jürimäe T, Mangus B, von Duvillard S. (2011) Peripheral signals of energy homeostasis as possible markers of training stress in athletes: a review. Metabolism, 60, 335350.

70. Kanaley J, Weltman J, Pieper K, Weltman A, Hartman M. (2001) Cortisol and growth hormone responses to exercise at different times of day. J Clin Endocrinol Metabol, 86, 2881-2889.

71. Kellmann M, Günther K. (2000) Changes in stress and recovery in elite rowers during preparation for the Olympic games. Med Sci Sports Exerc, 32, 676-683.

72. Kellmann M, Kallus K. (2001). Recovery-Stress Questionnaire for Athletes. Human Kinetics: Champaign, IL.

73. Kentta G, Hassmen P. (1998) Overtraining and Recovery. Sports Med, 26(1), 1-16.

74. Kenttä G, Hassmén P, Raglin J. (2001) Overtraining and staleness in Swedish age-group athletes: association with training behavior and psychosocial stressors. Intern J Sports Med, 22, 460-465.

75. Kenttä G, Hassmén P, Raglin J. (2006) Mood state monitoring of training and recovery in elite kayakers. Eur J Sport Sci, 4, 245-253.

76. Kern, W., Perras, B., Wodick, R., Fehm, H. L., and Born, J. (1995) Hormonal secretion during nighttime sleep indicating stress of daytime exercise. J Appl Physiol, 79, 1461-1468.

77. Kingsbury K, Kay L, Hjelm M. (1998) Contrasting plasma amino acid patterns in elite athletes: association with fatigue and infection. Br J Sports Med, 32, 25-33.

78. Kingsbury K, Kay L, Hjelm M. (1998) Contrasting plasma amino acid patterns in elite athletes: association with fatigue and infection. Br J Sports Med, 32, 25-33.

79. Kirwan J, Costill D, Mitchell J, Houmard J, Flynn M, Fink W, Beltz J. (1988) Carbohydrate balance in competitive runners during successive days of intense training. J. Appl. Physiol, 65(6), 26012606.

80. Kreider R, Fry AC, O'Toole M. (1998) Overtraining in sport: terms, definitions, and prevalence. In: Kreider R, Fry AC, O’Toole M, editors. Overtraining in sport. Champaign (IL): Human Kinetics, viiix 
81. Kuipers H, Keizer H. (1988) Overtraining in elite athletes, Sports Med. , 6, 79-92.

82. Kuipers H. (1996) How much is too much? Performance aspects of overtraining. Res $Q$ Exerc Sport , 67(s3), 65-69.

83. Lachuer J, Delton I, Buda M, Tappaz M. (1994) The habituation of brainstem catecholaminergic groups to chronic daily restraint stress is stress specific like that of the hypothalamo-pituitaryadrenal axis. Brain Res., 638, 196-202.

84. Lamberts R, Swart J, Capostagno B, Noakes T, Lambert M. (2010) Heart rate recovery as a guide to monitor fatigue and predict changes in performance parameters. Scand J Med Sci Sports Exerc, 20, 449-457.

85. Lancaster G, Halson S, Khan Q, Drysdale P, Jeukendrup A, Drayson M, Gleeson M. (2004) The effects of acute exhaustive exercise and intensified training on type 1/type $2 \mathrm{~T}$ cell distribution and cytokine production. Exerc Immunol Rev., 10, 91-106.

86. Lancaster G, Halson S, Khan Q, Drysdale P, Wallace F, Jeukendrup A, Drayson M, Gleeson M. (2003) Effect of acute exhaustive exercise and a 6-day period of intensified training on immune function in cyclists. J Physiol, 548.P, O96.

87. Lehmann M, Foster C, Dickhuth HH, Gastmann U. (1998) Autonomic imbalance hypothesis and overtraining syndrome. Med Sci Sports Exerc 30, 1140-1145.

88. Lehmann M, Foster C, Keul J. (1993b) Overtraining in endurance athletes: a brief review. Med Sci Sports Exerc, 25(7), 854-862.

89. Lehmann M, Knizia K, Gastmann U, Petersen KG, Khalaf AN, Bauer S, Kerp L, Keul J. (1993a) Influence of 6-week, 6 days per week, training on pituitary function in recreational athletes. $\mathrm{Br} J$ Sports Med, 27, 186-192.

90. Lehmann M, Petersen KG, Liu Y, Gastmann U, Lormes W, Steinacker JM. (2001) Chronische und erschöpfende Belastungen im Sport - Einfluss von Leptin und Inhibin. [Chronic and exhausting training in sports - influence of leptin and inhibin] Dtsch Z Sportmed, 51, 234 - 243.

91. Lemon P, Mullin. (1980) Effect of initial muscle glycogen on protein catabolism during exercise. $J$ Appl Physiol, 48, 624-629.

92. Mackinnon L, Hooper S, Jones S, Gordon R, Bachmann A. (1997) Hormonal, immunological, and haematological responses to intensified training in elite swimmers. Med Sci Sports Exerc, 29, 1637-1645.

93. Mackinnon L, Hooper S. (1994) Mucosal (secretory) immune system responses to exercise of varying intensity and during overtraining. Int J Sports Med, 15, S179-S183.

94. Matos N, Winsley R, Williams C. (2011) Prevalence of Nonfunctional Overreaching/Overtraining in Young English Athletes. Med Sci Sports Exerc, 43, 1287-1294.

95. McKenzie D. (1999) Markers of excessive exercise. Can J Appl Physiol, 24(1), 66-73.

96. Meeusen R, Duclos M, Gleeson M, Rietjens G, Steinacker J, Urhausen A. (2006) Prevention, diagnosis and treatment of the Overtraining Syndrome - ECSS Position Statement 'Task Force' Eur J Sport Sci, 6(1), 1-14.

97. Meeusen R, Nederhof E, Buyse L, Roelands B, De Schutter G, Piacentini MF. (2010) Diagnosing overtraining in athletes using the two bout exercise protocol. Br J Sports Med, 44 (9), 642-648.

98. Meeusen R, Piacentini MF, Busschaert B, Buyse L, De Schutter G, Stray-Gundersen J. (2004) Hormonal responses in athletes: the use of a two bout exercise protocol to detect subtle differences in (over)training status. Eur J Appl Physiol., 91, 140-146.

99. Meeusen R, Watson P. (2007) Amino acids and the brain: do they play a role in "central fatigue"? Int J Sport Nutr Exerc Metab, 17, S37-S46. 
100. Meeusen R. (1998) Overtraining, indoor \& outdoor. Vlaams tijdschrift voor Sportgeneeskunde \& Sportwetenschappen, 19, 8-19.

101. Moore C, Fry A, Harber M, Smith J, Rubin M. (2007) Nonfunctional overreaching during offseason training for skill position players in collegiate American football. J Strength Condition Res, 21(3), 793-800.

102. Morgado J, Rama L, Silva I, de Jesus Inácio M, Henriques A, Laranjeira P, Pedreiro S, Rosado F, Alves F, Gleeson M, Pais M, Paiva A, Teixeira A. (2011). Cytokine production by monocytes, neutrophils, and dendritic cells is hamperd by long-term intensive training in eleite swimmers. Eur J Appl Physiol, 112(2), 471-82, 2012.

103. Morgan W, Brown D, Raglin J, O'Connor P, Ellickson K. (1987a) Psychological monitoring of overtraining and staleness. Br J Sports Med, 21, 107-114.

104. Morgan W, Costill D, Flynn M, Raglin J, O'Connor P. (1988a) Mood disturbance following increased training in swimmers, Med Sci Sports Exerc, 20, 408-414.

105. Morgan W, O'Connor P, Ellickson K, Bradley P. (1988b) Personality structure, mood states, and performance in elite male distance runners. Intern J Sport Psychol, 19, 247-263.

106. Morgan W, O'Connor P, Sparling P, Pate R. (1987b) Psychological characterization of the elite female distance runner. Intern J Sports Med 8 (suppl.), 124-131.

107. Nederhof E, Lemmink K, Visscher C, Meeusen R, Mulder T. (2006) Psychomotor speed: possibly a new marker for overtraining syndrome. Sports Med, 36(10), 817-828.

108. Nederhof E, Lemmink K, Zwerver J, Mulder T. (2007) The effect of high load training on psychomotor speed. Int J Sports Med., 28(7), 595-601.

109. Nederhof E, Zwerver J, Brink M, Meeusen R, Lemmink K. (2008) Different diagnostic tools in nonfunctional overreaching. Int J Sports Med., 29(7), 590-597.

110. Neville V, Gleeson M, Folland J. (2008) Salivary IgA as a risk factor for upper respiratory infections in elite professional athletes. Med Sci Sports Exerc, 40, 1228-1236.

111. Nieman D. (1997) Immune response to heavy exertion. J Appl Physiol, 82, 1385-1394.

112. Nieman D. (2009) Immune function responses to ultramarathon race competition. Med Sportiva, 13, 1890196.

113. O'Connor P, Morgan W, Raglin J, Barksdale C, Kalin N. (1989) Mood state and salivary cortisol levels following overtraining in female swimmers. Psychoneuroendocr, 14, 303-310.

114. O'Connor P. (1997) Overtraining and staleness. In: W.P. Morgan (ed). Physical activity \& Mental Health. Taylor \& Francis, Washington, USA, pp 145-160.

115. Purvis D, Gonsalves S, Deuster P. (2010) Physiological and psychological fatigue in extreme conditions: overtraining and elite athletes. Phys Med Rehabil, 2, 442-450.

116. Raglin J, Morgan W, Luchsinger A. (1990) Mood state and self-motivation in successful and unsuccessful women rowers. Med Sci Sports Exerc, 22, 849-853.

117. Raglin J, Morgan W, O'Connor P, (1991) Changes in mood state during training in female and male college swimmers. Int J Sports Med, 12, 585-589 .

118. Raglin J, Morgan W. (1994) Development of a scale for use in monitoring training-induced distress in athletes. Int J Sports Med, 15, 84-88.

119. Raglin J, Sawamura S, Alexiou S, Hassmén P, Kenttä G. (2000) Training practices and staleness in 13-18 year old swimmers: A cross-cultural study. Pediatr Sports Med, 12, 61-70.

120. Raglin J, Wilson G. (2000). Overtraining and staleness in athletes. In: Y.L. Hanin (Ed.). Emotions in Sports (pp.191-207). Champaign, IL: Human Kinetics. 
121. Raglin J. (1993). Overtraining and staleness: Psychometric monitoring of endurance athletes. In: R.N. Singer, M. Murphey, \& L.K. Tennant (Eds.), Handbook of Research on Sport Psychology. (pp.840-850). New York: Macmillan.

122. Reid V, Gleeson M, Williams N, Clancy R. (2004) Clinical investigation of athletes with persistent fatigue and/or recurrent infections. Br J Sports Med, 38, 42-45.

123. Rietjens G, Kuipers H, Adam J, Saris W, Breda van E, Hamont van D, Keizer H. (2005) Physiological, biochemical and psychological markers of overreaching. Int J Sport med, 26, 16-26.

124. Robson P, Blannin A, Walsh N. (1999) The effect of an acute period of intense interval training on human neutrophil function and plasma glutamine in endurance-trained male runners. $J$ Physiol, 515, 84-85P.

125. Rowbottom D, Keast D, Goodman C, Morton A. (1995) The haematological, biochemical and immunological profile of athletes suffering from the Overtraining Syndrome. Eur J Appl Physiol, 70, 502-509.

126. Rushall, B. (1990) A tool for measuring stress tolerance in elite athletes. J App/ Sport Psychol, 2, 51-66.

127. Selye H. (1936) A syndrome produced by diverse nocuous agents. Nature, 138, 32.

128. Shephard R. (2001) Chronic fatigue syndrome: an update. Sports Med., 31(3), 167-194.

129. Shintani F, Nakaki T, Kanba S, Sato K, Yagi G, Shiozawa M, Aiso S, Kato R, Asai M. (1995) Involvement of interleukin-1 in immobilisation stress-induced increase in plasma adrenocorticotropic hormones and in release of hypothalamic monoamines in rat. J Neurosci, 15, 1961-1970.

130. Simsch C, Lormes W, Petersen KG, Liu Y, Hackney AC, Lehmann M, Steinacker JM. (2002) Training intensity influences leptin and thyroid hormones in highly trained rowers. Int J Sports Med, 23, 422-427.

131. Smith D, Norris S. (2000) Changes in glutamine and glutamate concentrations for tracking training tolerance. Med Sci Sports Exerc, 32(3), 684-689.

132. Smith L. (2000) Cytokine hypothesis of overtraining: a physiological adaptation to excessive stress? Med Sci Sports Exerc ,32, 317-331.

133. Snyder A, Jeukendrup A, Hesselink M, Kuipers H, Foster C. (1993) A physiological/psychological indicator of overreaching during intensive training. Int J Sports Med, 14, 29-32.

134. Snyder A. (1999) Overtraining and glycogen depletion hypothesis. Med Sci Sports Exerc, 30, $1146-1150$

135. Snyder A. (1999). Overtraining and glycogen depletion hypothesis. Med Sci Sports, Exerc, 30, 1146-1150.

136. Steinacker J , Lormes W, Liu Y, Opitz-Gress A, Baller B, Günther K, Gastmann U, Petersen KG, Lehmann M, Altenburg D. (2000) Training of Junior Rowers before World Championships. Effects on performance, mood state and selected hormonal and metabolic responses. $J$ Phys Fit Sports Med, 40, 327-335.

137. Steinacker J, Lormes W, Reissnecker S, Liu Y. (2004) New aspects of the hormone and cytokine response to training. Eur J Appl Physiol ,91, 382-93.

138. Tergau F, Geese R, Bauer A, Baur S, Paulus W, Reimers CD. (2000) Motor cortex fatigue in sports measured by transcranial magnetic double stimulation. Med Sci Sports Exerc., 32(11), 19421948. 
139. Tiollier E, Gomez-Merino D, Burnat P, Jouanin JC, Bourrilhon C, Filaire E, Guezennec CY, Chennaoui M. (2005) Intense training: Intense training: mucosal immunity and incidence of respiratory infections. Eur J Appl Physiol, 93, 421-428.

140. Urhausen A, Gabriel H, Kindermann W. (1995) Blood hormones as markers of training stress and overtraining. Sports Med., 20, 251-276.

141. Urhausen A, Gabriel H, Kindermann W. (1998a) Impaired pituitary hormonal response to exhaustive exercise in overtrained endurance athletes. Med Sci Sports Exerc., 30, 407-414.

142. Urhausen A, Gabriel H, Weiler B, Kindermann W. (1998b) Ergometric and psychological findings during overtraining: a long-term follow-up study in endurance athletes. Int J Sports Med, 19, 114-120.

143. Urhausen A, Kindermann W. (2002) Diagnosis of Overtraining - What Tools do we have ? Sports Med, 32, 95-102.

144. Urhausen A, Kindermann W. (1992) Biochemical monitoring of training. Clin J Sports Med, 2, 5261.

145. Uusitalo A, Uusitalo A, Rusko H. (1998) Exhaustive endurance training for 6-9 weeks did not induce changes in intrinsic heart rate and cardiac autonomic modulation in female athletes. Int $J$ Sports Med, 19, 532-540.

146. Uusitalo A, Uusitalo A, Rusko H. (2000) Heart rate and blood pressure variability during heavy training and overtraining in the female athlete. Int J Sports Med, 21,45-53.

147. Uusitalo A. (2001) Overtraining. Making a difficult diagnosis and implementing targeted treatment. The Physician and Sportsmedicine, 29, 35-50.

148. Verde T, Thomas S, Shephard RJ. (1992) Potential markers of heavy training in highly trained endurance runners. Brit J Sports Med, 26, 167-175.

149. Walsh N, Gleeson M, Shephard R, Gleeson M, Woods J, Bishop N, Fleshner M, Green C, Pedersen B, Hoffman-Goetz L, Rogers C, Northoff H, Abbasi A, Simon P. (2011) Position Statement Part One: Immune function and exercise. Exerc Immunol Rev, 17, 6-63.

150. Walsh, N, Blannin A, Robson P, Gleeson M. (1998) Glutamine, exercise and immune function: links and possible mechanisms. Sports Med , 26, 177-191.

151. Wilson G, Raglin J. (2004) The predictive value of hardiness and optimism for theidentification of past staleness responses in high school age male and female distance runners. New Studies in Athletics, 19, 41-46.

152. Wittert G, Livesey J, Espiner E, Donald R. (1996) Adaptation of the hypothalamo-pituitary adrenal axis to chronic exercise stress in humans. Med Sci Sports Exerc. , 28, 1015-1019.

Acknowledgement :

The assistance of Martina Velders and Benjamin Koch, University of ULM, Germany is much appreciated. 
(17-07-2012) 